\title{
Effects of Taiji Practice on Mindfulness and Self-Compassion in Healthy Participants-A Randomized Controlled Trial
}

\author{
Marko Nedeljkovic • Petra H. Wirtz • \\ Brigitte Ausfeld-Hafter
}

Published online: 26 February 2012

(C) Springer Science+Business Media, LLC 2012

\begin{abstract}
Taiji is regarded as a mind-body practice that is characterized by gentle and mindful body movements. In contrast to the continuously growing evidence base supporting the beneficial effects of Taiji on physical and mental well-being, studies investigating its underlying mechanisms are still scarce. The aim of our study was to examine the impact of Taiji practice on self-attribution of mindfulness and self-compassion, two potential components well known for their health promoting effects. Seventy healthy participants (age range: 23-50 years) were randomly assigned either to the intervention group or to a wait list control group. The intervention group attended Taiji classes twice a week for 3 months. Before, shortly after and 2 months after the intervention, we measured the degree of selfattributed mindfulness and self-compassion in all study participants by using self-report questionnaires. Compared to the control group, the intervention group showed significantly higher increase scores in self-attributed mindfulness after the intervention that persisted 2 months later. Increases in self-attributed self-compassion were also higher in Taiji practitioners, with significant group differences from preintervention to follow-up assessment. Our findings suggest that Taiji practice can effectively enhance self-attribution of mindfulness and is likely to have beneficial effects on
\end{abstract}

\footnotetext{
M. Nedeljkovic $(\triangle) \cdot$ B. Ausfeld-Hafter

Institute of Complementary Medicine, University of Bern, Imhoof-Pavillon, Inselspital,

CH-3010 Bern, Switzerland

e-mail: marko.nedeljkovic@kikom.unibe.ch

\section{P. H. Wirtz}

Institute of Psychology,

Department of Biological and Health Psychology,

University of Bern,

Bern, Switzerland

self-compassion in healthy participants. The role of mindfulness as a mechanism underlying the beneficial effects of Taiji practice warrants further research.

Keywords Taiji $\cdot$ Mindfulness · Self-compassion · Mind-body $\cdot$ Randomized controlled trial

\section{Introduction}

In recent years, mind-body practices have received considerable attention in the general population, both among patients and in the research community (Barnes et al. 2004; Bertisch et al. 2009; Cullen 2011; Michalsen 2010; Ospina et al. 2008). Mind-body practices cover a wide range of healing practices, sharing the common intention to use the mind to affect physical functioning and promote health (National Institutes of Health 2010). Taiji-variably spelled Taijiquan, Tai Chi or Tai Chi Chuan-is one of them. It is a form of slow intentional body movements rooted in ancient Chinese martial arts (e.g., Robinson 2006; Wayne and Kaptchuk 2008a). This mind-body practice "... aims to strengthen and relax the physical body and mind, enhance the natural flow of what the Chinese call qi (a nontranslatable word that describes ... life energy), and improve health, personal development, and self-defense" (Wayne and Kaptchuk 2008a, p.96). In fact, a growing body of evidence supports the preventive and therapeutic values of Taiji (Jahnke et al. 2010; Klein and Adams 2004; Nedeljkovic et al. 2012; Wang et al. 2010; Wang 2011). However, studies investigating how this mind-body practice might work are scarce. Mindfulness has been suggested as a component that potentially mediates the beneficial effects of Taiji practice (Esch et al. 2007; Wayne and Kaptchuk 2008a). 
Historically, mindfulness is closely related to meditation practice aiming at cultivating nonjudgmental awareness of one's moment-by-moment experience (Kabat-Zinn 2003). Mindfulness has been operationalized as a multifaceted construct including the ability to observe and to describe experiences, to act with awareness of the present moment and to maintain a nonjudgmental and nonreactive attitude towards inner experience (Baer et al. 2006). Interventions based on mindfulness have repeatedly been shown to be effective in treating a variety of physical and mental disorders (Baer 2003; Grossman et al. 2004; Hofmann et al. 2010; Praissman 2008). The assumption that practicing Taiji might increase mindful awareness of one's bodily sensations is supported by findings reporting an increase of body awareness (Gyllensten et al. 2010; Uhlig et al. 2010) and kinetic sense (Jacobson et al. 1997) due to Taiji practice. The influence of Taiji practice on self-attributed mindfulness in a broader context has been examined by Caldwell et al. (2010). A significant increase in overall mindfulness scores as well as in the subscale scores for observing experience, nonjudging of and nonreacting to inner experience was observed in healthy college students after completion of a 15 -week Taiji course (Caldwell et al. 2010). Similarly, Zahn (2009) investigated the effects of Taiji on self-attribution of mindfulness in ten adolescent girls. Although some improvements in mindfulness scores were found, the results were reported as inconclusive. Notably, both of these studies did not include a control group, and there was no follow-up assessment of Taiji effects on mindfulness. Hitherto, studies investigating the role of mindfulness in Taiji practice that include a randomized controlled design and a follow-up period have not yet been reported.

Another concept closely linked to mindfulness is selfcompassion. Self-compassion is regarded as the ability to refer to oneself kindly when encountering distressing circumstances (Neff 2003a, b). The conceptualization of self-compassion according to Neff (2003a, b) comprises three interacting and overlapping components: "self-kindness," which represents an attitude of kindness, understanding and care towards oneself instead of harsh self-judgment and self-criticism. "Common humanity," which is regarded as the ability to see one's experience as part of the shared human experience, so that one feels connected rather than isolated from others. "Mindfulness," which represents the ability to remain mindfully aware of one's painful experiences rather than overidentifying with them. Previous research has shown that higher levels of self-compassion are associated with various favorable conditions such as greater life satisfaction, happiness, personal initiative, social connectedness and positive affect as well as less depression, anxiety, perfectionism, selfcriticism and disordered eating behaviors (for a recent review see Neff (2009)). Self-compassion can, therefore, be considered a health protective factor fostering emotional resilience
(Neff 2009). Interestingly, recent findings support the role of self-compassion as an important mediator in mindfulnessbased interventions (Birnie et al. 2010; Kuyken et al. 2010; Shapiro et al. 2005). Given this reasoning, and given that Taiji may enhance self-attributed mindfulness (Caldwell et al. 2010), self-compassion might be cultivated through Taiji practice. However, self-compassion has not yet been examined as a potential component inherent to Taiji practice.

In sum, self-attributed mindfulness and self-compassion are two interrelated key components of mindfulness-based interventions, both known for their health promoting effects. However, their role in Taiji practice remains to be elucidated. We thus set out to investigate whether Taiji practice enhances self-attribution of mindfulness and self-compassion in a randomized-controlled study design with a 2-month followup period.

\section{Methods}

\section{Participants and Design}

This study was part of a trial examining psychobiological effects of Taiji on psychosocial stress reactivity (Nedeljkovic et al. 2012) and was formally approved by the ethics committee of the Canton of Bern, Switzerland. Study participants were recruited through advertisement of the study on pin boards and on the homepage at the University of Bern, and at the University Hospital in Bern.

Applicants underwent a telephone screening and were included in the study if they were older than 18 years and if the following exclusion criteria did not apply: regular or occasional intake of prescription drugs, acute or chronic physical or mental disorders, pregnancy, consumption of tobacco (more than five cigarettes per day), consumption of alcoholic drinks (more than two drinks per day), consumption of psychoactive substances, more than 1 week of absence during the Taiji course or previous practical experience with Taiji. Complete written and oral description of the study was provided to all included participants, and informed written consent was obtained prior to participation. Following the preintervention assessment, participants were randomly assigned either to the Taiji group or to the wait list control group. Allocation concealment was achieved by using sequentially numbered, opaque and sealed envelopes. They were opened individually for each eligible participant. The group characteristics are presented in Table 1.

\section{Taiji Intervention}

The Taiji course lasted for 12 weeks. Training sessions took place twice a week and lasted for $60 \mathrm{~min}$ each. Participants who missed a training session were offered extra classes to 
Table 1 Group characteristics and baseline values

\begin{tabular}{|c|c|c|c|}
\hline Group characteristics ${ }^{\mathrm{a}}$ & Taiji group $(n=28)$ & Control group $(n=31)$ & $p$ \\
\hline Age (years) & $35.86 \pm 1.63$ & $35.13 \pm 1.35$ & 0.74 \\
\hline Gender (male/female) & $10 / 18$ & $10 / 21$ & 0.79 \\
\hline Body mass index $\left(\mathrm{kg} / \mathrm{m}^{2}\right)$ & $23.38 \pm 0.63$ & $23.11 \pm 0.79$ & 0.79 \\
\hline Education (with/without high school degree, i.e., Swiss "Matura") & $21 / 7$ & $25 / 6$ & 0.76 \\
\hline Occupational status (full or part time workers/students) & $25 / 3$ & $30 / 1$ & 0.34 \\
\hline Smoking (nonsmokers/light smokers) & $23 / 5$ & $24 / 7$ & 0.75 \\
\hline Previous experience with mind-body practices (months of regular practice) & $15.79 \pm 5.67$ & $30.04 \pm 10.18$ & 0.21 \\
\hline Taiji classes attended (incl. \%-value) & $20.8 \pm 0.56(86.8 \%)$ & - & - \\
\hline \multicolumn{4}{|l|}{ Baseline values } \\
\hline Mindfulness (FMI-SF total score) & $38.96 \pm 0.86$ & $34.74 \pm 0.94$ & 0.002 \\
\hline Self-compassion (SCS total score) & $3.31 \pm 0.087$ & $3.02 \pm 0.094$ & 0.032 \\
\hline —Self-kindness (SCS subscale score) & $3.49 \pm 0.099$ & $2.99 \pm 0.118$ & 0.002 \\
\hline —Self-judgment (SCS subscale score) & $2.81 \pm 0.123$ & $3.02 \pm 0.095$ & 0.17 \\
\hline —Common humanity (SCS subscale score) & $3.12 \pm 0.159$ & $2.90 \pm 0.139$ & 0.30 \\
\hline —Isolation (SCS subscale score) & $2.46 \pm 0.153$ & $2.60 \pm 0.147$ & 0.49 \\
\hline —Mindfulness (SCS subscale score) & $3.45 \pm 0.103$ & $3.23 \pm 0.124$ & 0.20 \\
\hline —Overidentification (SCS subscale score) & $2.96 \pm 0.129$ & $3.36 \pm 0.125$ & 0.027 \\
\hline
\end{tabular}

${ }^{\mathrm{a}}$ Continuous data are expressed as mean \pm S.E.M.

${ }^{\mathrm{b}} p$-Values refer to $\chi^{2}$ tests for categorical data and independent samples $t$-tests for continuous data

compensate, and additional Taiji practice at home was encouraged in the intervention group. Participants' class attendance was recorded by the Taiji teacher, who was in charge of all classes. The Taiji teacher is certified by the Swiss Society for Qigong and Taijiquan (Schweizerische Gesellschaft für Qigong und Taijiquan-SGQT). Participants were taught the first 18 sequences of the 37 Yang style Taiji short form (e.g., Robinson 2006). The focus in the Taiji course was laid on basic principles which are common to all Taiji styles such as extension, relaxation and alignment of the body as well as mindful and holistic body movements (Wolf et al. 1997). Each Taiji session started with warm up exercises, followed by practicing Taiji movements and reviewing Taiji principles, and ended with Taiji-related breathing and relaxation exercises. All participants were instructed not to join any other new mind-body or physical exercise program during their study participation. After study termination, an equivalent Taiji course was offered to all participants in the control group.

\section{Measures}

\section{Freiburg Mindfulness Inventory-Short Form (FMI-SF)}

The FMI-SF consists of 14 items, all measured on a Likert scale ranging from 1 (rarely) to 4 (almost always). A good internal consistency has been reported for the general factor (Cronbach's alpha $=0.79$ to 0.86 ). Due to considerable second loadings of the items, self-attributed mindfulness, as it is measured by the FMI-SF, is preferably regarded as a general construct that has interrelated facets. Its items are considered to represent the core of the mindfulness construct. As specific knowledge of the Buddhist background of mindfulness is not required to fill out the inventory, the authors recommend its use for participants without previous meditation experience (Walach et al. 2006).

\section{Self-Compassion Scale (SCS)}

The SCS was developed by Neff (2003b) as a 26-item selfreport measure. In accordance with this original version, the validated German translation of the SCS (Hupfeld and Ruffieux 2011), which has been used in our study, is also assessing the following six subscales: self-kindness $(\alpha=$ $0.83)$, self-judgment ( $\alpha=0.74)$, common humanity ( $\alpha=$ $0.75)$, isolation $(\alpha=0.80)$, mindfulness $(\alpha=0.66)$ and overidentification $(\alpha=0.70)$. All subscales together constitute the total self-compassion scale $(\alpha=0.91)$. Each item is scored on a Likert scale ranging from 1 (almost never) to 5 (almost always).

\section{Procedure}

At preintervention assessment, participants completed an online survey comprising the SCS and the FMI-SF questionnaires, alongside other questionnaires not relevant to the present study. All study participants were asked to complete 
postintervention assessment after the last Taiji class (week 12) and follow-up assessment 2 months after the intervention ended (week 20). Participants not responding within 5 days were reminded by phone call and email.

\section{Statistical Analysis}

Data analysis was conducted by using SPSS (version 18) statistical software package for Macintosh (IBM SPSS Statistics. Somers, NY, USA). The optimal total sample size was calculated with the statistical software $\mathrm{G}^{*}$ Power (Faul et al. 2007). To detect an expected effect size of $f=0.35$ (representing a medium to large effect size in $F$ tests) with a power $\geq 0.80$ and $\alpha=0.05$, the optimal total sample size of $n=68$ was calculated a priori, which is in line with previously reported sample size recommendations for studies in mindfulness research (Baer 2003). Effect size parameters ( $f$ ) were calculated from partial $\eta^{2}$ values and are reported where appropriate (effect size conventions: $f: 0.10=$ small, $0.25=$ medium, $0.40=$ large; see Cohen (1988)). All analyses were two-tailed, with the level of significance set at $p<0.05$. Unless indicated, all results are presented as mean \pm standard error of means (S.E.M.). Prior to statistical analyses, all data were tested for normal distribution and homogeneity of variance using a Kolmogorov-Smirnov and Levene test. Group characteristics were analyzed by $\chi^{2}$ analysis for categorical data and independent samples $t$-test for continuous data. Group differences in baseline values were also tested using $t$-tests. For participants lost to follow-up, their last value was carried forward.

To test whether Taiji practice enhances self-attributed mindfulness and self-compassion, we calculated repeated measures ANCOVAs with group (Taiji vs. control) as the independent variable and repeated measures of selfattributed mindfulness and self-compassion, respectively, as dependent variables. As baseline values of selfattributed mindfulness and self-compassion significantly differed between the two study groups and since we were interested in intervention-induced changes, all dependent variables were corrected for the respective baseline levels that were entered as covariates in all analyses. Posthoc analyses were conducted to test whether the study groups significantly differed in their intervention-related change scores (Taiji vs. waiting) by calculating one-way ANCOVAs with group as the independent variable and pre-postintervention changes as well as changes from preintervention to follow-up assessment as dependent variables. Again, baseline values were considered as covariates.

To test the validity of the employed questionnaires, we conducted exploratory analyses by calculating Pearson's correlation coefficients between the FMI-SF scale and the sum scale of SCS as well as the three SCS subscales for all three time points.

\section{Results}

Of the 112 applicants who underwent a telephone screening, 70 participants fulfilled inclusion criteria and successfully completed preintervention assessment. They were randomly assigned to either the Taiji group $(n=35)$ or the wait list control group $(n=35)$. As 11 participants discontinued (Taiji group $n=7$ : two never attended the Taiji course, four had scheduling conflicts, one was injured due to an accident/ control group $n=4$ : three lost interest in participation right after group assignment, one moved out of Bern area), postintervention measures were obtained from 28 participants of the Taiji group and 31 participants of the control group (see Fig. 1). For unknown reasons, one participant of the Taiji group and one participant of the control group were not available for follow-up assessment.

\section{Group Characteristics}

The two study groups did not significantly differ in terms of age, gender, body mass index, education, occupational status, smoking or previous practical experience with selfapplicable mind-body practices (see Table 1). At baseline, the Taiji group reported significantly higher levels of mindfulness $(t=3.28, \mathrm{df}=57, p=0.002)$, general self-compassion $(t=2.20, \mathrm{df}=57, p=0.032)$ as well as higher scores on the SCS subscale "self-kindness" $(t=3.20, \mathrm{df}=57, p=0.002)$ and lower scores on the SCS subscale "overidentification" $(t=-2.72, \mathrm{df}=57, p=0.027)$ (see Table 1). Dropout participants did not significantly differ from the participants completing the study in any group characteristic (data not shown; $p$ 's $>0.17$ ).

\section{Taiji Effects on Self-attribution of Mindfulness} and Self-Compassion

Repeated measures ANCOVAs revealed higher selfattributed mindfulness and self-compassion scores before and after the intervention (Taiji vs. waiting) (main effect group: mindfulness: $F(1 / 56)=10.32, p=0.002, f=0.43$; selfcompassion: $F(1 / 56)=7.68, p=0.008, f=0.37)$ as well as in reaction to the intervention (interaction group by time: mindfulness: $F(1.94 / 108.72)=4.18, p=0.019, f=0.27$; selfcompassion: $F(2 / 112)=6.77, p=0.002, f=0.35)$ in the Taiji group as compared to the control group.

Posthoc testing of mindfulness revealed significantly higher increase scores in self-attributed mindfulness as measured by the FMI, from pre- to postintervention assessment $(F(1 / 56)=4.29, p=0.043)$ compared to controls. Similarly, follow-up measures indicate continuously increased selfattributed mindfulness scores in Taiji practitioners $(F(1 / 56)=$ 10.90, $p=0.002$; see Fig. 2a). Raw data, $p$-values and effect sizes are listed in Table 2. 


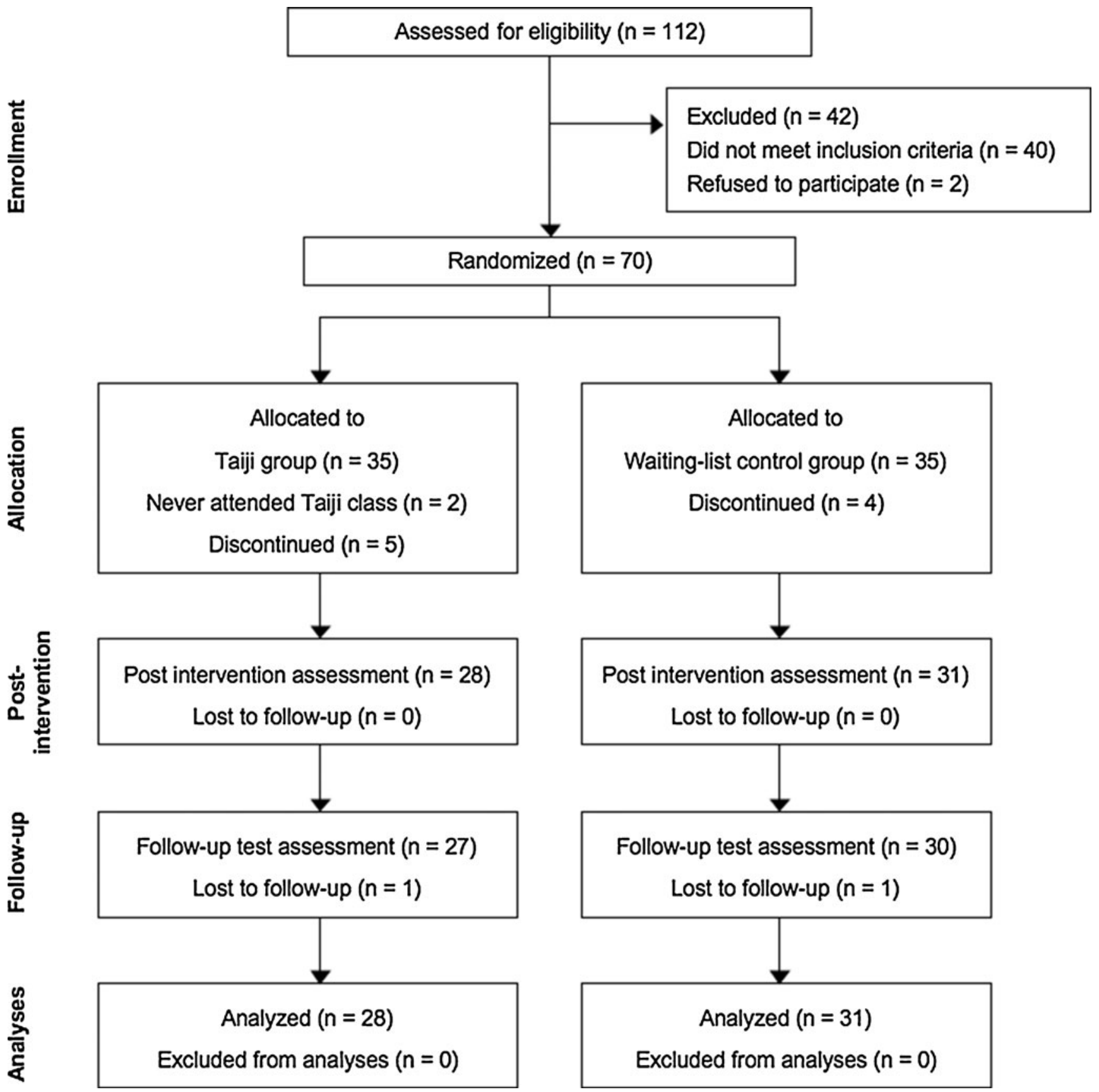

Fig. 1 CONSORT flow diagram depicting the progress through the phases of the randomized trial

Posthoc testing of self-compassion revealed higher SCS increase scores in the Taiji group as compared to the control group from preintervention to follow-up $(F(1 / 56)=12.54$, $p=0.001$; see Fig. 2b). Further testing of SCS subscales confirmed higher increase scores in the Taiji group in terms of self-kindness $(F(1 / 56)=7.37, p=0.009)$, common humanity $(F(1 / 56)=4.06, p=0.049)$ and mindfulness $(F(1 / 56)=$ $8.13, p=0.006)$, while higher decrease scores were observed for self-judgment $(F(1 / 56)=9.28, p=0.004)$ and isolation $(F(1 / 56)=8.68, p=0.005)$. No group differences in any
SCS change scores were observed from pre- to postintervention assessment, except for the SCS mindfulness subscale $(F(1 / 56)=5.94, p=0.018)$.

To explore whether Taiji practice exerts a stronger impact on the acceptance or on the presence facet of self-attributed mindfulness, we calculated and compared sum scores of specific FMI items that have been associated with the two facets "acceptance" and "presence" (Kohls et al. 2009). Compared to controls, participants in the Taiji group had significantly higher baseline values in FMI acceptance 


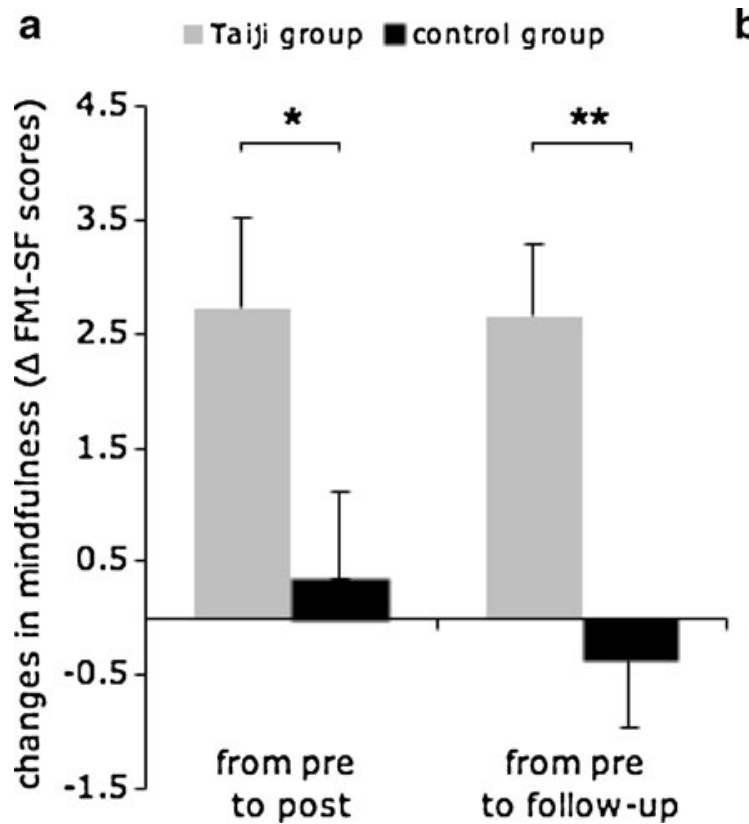

Fig. 2 Changes from pre- to postintervention assessment as well as from preintervention to follow-up assessment are depicted for selfattributed mindfulness scores in a and for self-compassion scores in b. Values are estimated marginal means \pm standard error of means (S.E. b

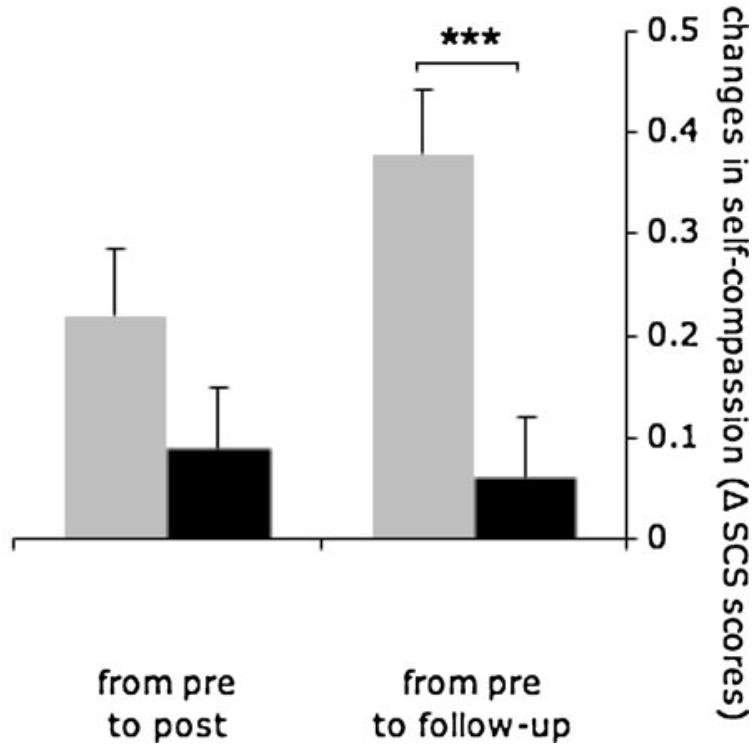

M.) and refer to ANCOVA's controlling for baseline values. Significance levels are indicated by an asterisk $(p<0.05)$, two asterisks $(p<0.01)$ and three asterisk $(p<0.001)$. FMI-SF Freiburg Mindfulness InventoryShort Form, SCS Self-Compassion Scale scores $(M=10.93 \pm 1.59(\mathrm{SD})$ vs. $M=9.45 \pm 1.77(\mathrm{SD}) ; t=$ 3.37, $\mathrm{df}=57, p=0.001)$ and FMI presence $(M=11.79 \pm 2.08$ (SD) vs. $M=10.71 \pm 1.90(\mathrm{SD}) ; t=2.08, \mathrm{df}=57, p=0.042$ ). We, therefore, employed the same analytical procedure as described in "Methods". Repeated measures ANCOVAs revealed higher FMI acceptance and presence scores before and after the intervention (Taiji vs. waiting) (main effect group: FMI acceptance: $F(1 / 56)=5.61, p=0.021, f=0.32$; FMI presence: $F(1 / 56)=12.40, p<0.001, f=0.47$ ) as well as in reaction to the intervention (interaction group by time: FMI acceptance: $F(1.99 / 111.54)=3.14, p=0.047, f=0.24$; FMI presence: $F(1.88 / 105.50)=6.40, p=0.003, f=0.34)$ in the Taiji group as compared to the control group.

\section{Correlations Between Self-attributed Mindfulness and Self-Compassion}

Our exploratory analysis revealed high positive correlations of the FMI scale with all SCS scales at all three time points $(p$ 's $<0.01)$. In detail, FMI correlated with the SCS sum scale (baseline: $r=0.67$, post: $r=0.77$, follow-up: $r=0.68$ ), the SCS subscales "mindfulness" (baseline: $r=0.59$, post: $r=0.72$, follow-up: $r=0.64$ ), "self-kindness" (baseline: $r=0.73$, post: $r=0.74$, followup: $r=0.68$ ), and "common humanity" (baseline: $r=0.41$, post: $r=0.52$, follow-up: $r=0.55$ ).

\section{Discussion}

To our knowledge, this is the first randomized controlled trial to examine the impact of Taiji on self-attributed mindfulness and self-compassion. We found that 3 months of regular Taiji practice led to a significant increase of selfreported mindfulness in healthy Taiji beginners compared to the wait list control group, and follow-up assessment revealed constantly increased levels of mindfulness in the Taiji group. Furthermore, we observed a significant increase in self-compassion from preintervention to follow-up assessment in the Taiji group as compared to controls. The present results extend previous research by suggesting that Taiji practice persistently enhances mindfulness and may cultivate self-compassion with effect sizes ranging from medium to large.

We consistently observed higher increase scores in selfattributed mindfulness in the Taiji group as measured by the FMI-SF questionnaire and the SCS mindfulness subscale suggesting that Taiji practice enhances mindfulness. This result extends previously reported findings on selfattributed mindfulness increases following Taiji practice (Caldwell et al. 2010) by considering for the first time not only a wait list control group but also a 2-month follow-up.

To explain why Taiji is likely to enhance self-attributed mindfulness, some commonalities between this mind-body 
Table 2 Changes in self-attributed mindfulness and self-compassion scores
${ }^{a} p$-Values refer to ANCOVAs controlling for baseline values
Variable
Mean change from baseline $( \pm \mathrm{SD})$

Taiji group $(n=28)$
Control group $(n=31)$

\begin{tabular}{|c|c|c|c|c|}
\hline \multicolumn{5}{|l|}{ FMI score } \\
\hline -Week 12 (postintervention) & $+1.96 \pm 4.06$ & $+1.03 \pm 4.55$ & 0.043 & 0.28 \\
\hline —Week 20 (follow-up) & $+1.54 \pm 3.49$ & $+0.65 \pm 4.48$ & 0.002 & 0.44 \\
\hline \multicolumn{5}{|l|}{ SCS total score } \\
\hline -Week 12 & $+0.20 \pm 0.34$ & $+0.11 \pm 0.33$ & 0.16 & - \\
\hline -Week 20 & $+0.34 \pm 0.38$ & $+0.09 \pm 0.32$ & $<0.001$ & 0.47 \\
\hline \multicolumn{5}{|l|}{ SCS self-kindness score } \\
\hline —Week 12 & $+0.14 \pm 0.45$ & $+0.22 \pm 0.49$ & 0.75 & - \\
\hline —Week 20 & $+0.42 \pm 0.44$ & $+0.21 \pm 0.52$ & 0.009 & 0.36 \\
\hline \multicolumn{5}{|l|}{ SCS self-judgment score } \\
\hline -Week 12 & $-0.20 \pm 0.57$ & $-0.08 \pm 0.58$ & 0.19 & - \\
\hline -Week 20 & $-0.38 \pm 0.53$ & $-0.05 \pm 0.58$ & 0.004 & 0.41 \\
\hline \multicolumn{5}{|l|}{ SCS common humanity score } \\
\hline -Week 12 & $+0.25 \pm 0.56$ & $+0.08 \pm 0.60$ & 0.06 & - \\
\hline —Week 20 & $+0.22 \pm 0.66$ & $0.00 \pm 0.76$ & 0.049 & 0.27 \\
\hline \multicolumn{5}{|l|}{ SCS Isolation score } \\
\hline —Week 12 & $-0.20 \pm 0.73$ & $-0.04 \pm 0.55$ & 0.26 & - \\
\hline —Week 20 & $-0.40 \pm 0.74$ & $\pm 0.00 \pm 0.52$ & 0.005 & 0.39 \\
\hline \multicolumn{5}{|l|}{ SCS mindfulness score } \\
\hline —Week 12 & $+0.23 \pm 0.48$ & $-0.04 \pm 0.48$ & 0.018 & 0.33 \\
\hline -Week 20 & $+0.31 \pm 0.50$ & $+0.03 \pm 0.57$ & 0.006 & 0.38 \\
\hline \multicolumn{5}{|l|}{ SCS overidentification score } \\
\hline -Week 12 & $-0.19 \pm 0.72$ & $-0.25 \pm 0.49$ & 0.67 & - \\
\hline -Week 20 & $-0.31 \pm 0.56$ & $-0.26 \pm 0.49$ & 0.27 & - \\
\hline
\end{tabular}

practice and mindfulness-based interventions shall be briefly presented. Mindfulness is not only closely linked to Buddhist meditation practices but is also found in other spiritual and philosophical traditions such as Taoist philosophy (Kabat-Zinn 2003), which is commonly integrated in Taiji practice (e.g., Frantzis 1998; Robinson 2006). In our Taiji course, we focused on developing Taiji-related body awareness and body mechanics by conveying an embodiment of the basic Taiji principles, which are rooted in Taoist philosophy. For doing so, the Taiji teacher used a guided self-explorative teaching approach, similar to the introspective method applied in the body scan exercise in mindfulnessbased stress reduction (MBSR) programs. Notably, the cultivation of body awareness in stillness and motion is also emphasized in MBSR programs and other mindfulness based interventions, since the body is considered "as a doorway to the present moment" (Cullen 2011, p.192). In fact, our explorative analyses support the notion that Taiji practice has a significant impact on both, on the acceptance facet and, even more pronounced, on the presence facet of self-reported mindfulness. Furthermore, the embodiment of the essential course content by the teacher is regarded as the primary vehicle for teaching in Taiji classes as well as in mindfulness-based interventions (Cullen 2011; Wayne and Kaptchuk 2008a). Eventually, similar subjective outcomes (i.e., greater confidence, stress hardiness and greater ability to modulate emotional triggers) are proposed by both, the Taiji and the MBSR approach (Wall 2005). Importantly, one should be aware of the pluralism of Taiji. There are various recognized styles of Taiji, each one using a slightly different emphasis regarding training content as well as core Taiji principles (Frantzis 1998). Likewise, there is a large variety in the overall focus of Taiji training (e.g., health maintenance, martial skills, meditation and self-realization or competitive performing arts). The varying length of Taiji forms, distinctive teaching approaches (e.g., predominantly nonverbal or verbal instructions) and other aspects such as the variability in the format of training programs (i.e., group vs. individual training program) as well as in the training content (e.g., in- or exclusion of weapons training and interactive two-person exercises) are further adding to the significant heterogeneity in the training that Taiji students experience (Wayne and Kaptchuk 2008b). Therefore, Taiji cannot be fully compared with more standardized interventions such as the MBSR program. 
Interestingly, we could not detect group differences for self-compassion in the mean change values from pre- to postintervention assessment. It may be speculated that selfcompassion becomes more relevant for active self-care once the supportive setting of guided and regular Taiji classes is no longer provided. It is likely that this absence of regular Taiji classes demands a higher effort to devote time for practicing Taiji. As Bögels et al. (2010) proposed, it is due to this devotion of time to meditative practices that participants learn to draw positive attention to the self and, thus, become more compassionate towards themselves. For a better understanding of how self-compassion might be fostered through Taiji practice, further longitudinal studies comprising qualitative methods are needed.

By emphasizing the common features of Taiji practice and mindfulness-based interventions, we suggest that both approaches might be used together. Notably, a combination of Taiji exercises with MBSR in middle school children has previously been reported (Wall 2005). Whether participants who are not responding to mindfulness-based interventions might alternatively benefit from a primarily body-oriented approach like Taiji should be addressed in future research.

Our study has some limitations. First, despite randomized group allocation, we found significant baseline differences in self-attributed mindfulness and self-compassion between the two study groups. We could only control for this potentially confounding factor statistically. Although randomized group allocation is usually regarded as a well-accepted and useful allocation procedure, future studies may consider a baseline assessment-related group allocation to rule out baseline group differences. Second, as we measured selfattributed mindfulness using the FMI-SF inventory, we obtained a score representing the general level of selfattributed mindfulness. To get a more differentiated view on the Taiji-related effects on self-attributed mindfulness, we recommend the use of questionnaires assessing different facets of mindfulness as well as assessing third party ratings in future research. Third, as the focus of our study was laid on efficacy research, we did not include an active control group in our study design. Further studies engaging in comparative effectiveness research should comprise an exercise control group not associated with mindful body movements. Finally, the extent to which the findings obtained in our nonclinical sample can be generalized to clinical populations remains unclear.

The main strengths of this study are its randomized controlled design with a follow-up period of 2 months and the application of restrictive exclusion criteria, both contributing to a high internal validity. Moreover, the total sample size was large enough for meaningful power calculations, and our study sample allows for a high degree of generalizability in the healthy general population. Finally, we assessed the level of self-attributed mindfulness by using two different questionnaires (FMI-SF and SCS mindfulness subscale) and obtained consistent results as well as additional information about self-compassion.

In conclusion, our findings suggest that self-attributed mindfulness can be effectively increased, and self-compassion may be cultivated by practicing Taiji. Thus, mindfulness may be considered a relevant component inherent to Taiji practice. Its role as a potential mediator of beneficial effects induced by Taiji practice warrants further research.

Acknowledgments This trial was funded by Stiftung für Komplementärmedizin, Gottfried und Julia Bangerter-Rhyner Stiftung, Parrotia Stiftung (to $\mathrm{MN}$ ) and by the Swiss National Foundation Grant PP00P1_128565/1 (to PHW). The funding sources had no role in study design, in the collection, analysis and interpretation of data, in the writing of the report and in the decision to submit the paper for publication.

Conflict of interest All authors declare that they have no conflicts of interest.

\section{References}

Baer, R. A. (2003). Mindfulness training as a clinical intervention: A conceptual and empirical review. Clinical Psychology: Science and Practice, 10(2), 125-143.

Baer, R. A., Smith, G. T., Hopkins, J., Krietemeyer, J., \& Toney, L. (2006). Using self-reported assessment methods to explore facets of mindfulness. Assessment, 13(1), 27-45.

Barnes, P. M., Powell-Griner, E., McFann, K., \& Nahin, R. L. (2004). Complementary and alternative medicine use among adults: United States. Advance Data, 343, 1-19.

Bertisch, S. M., Wee, C. C., Phillips, R. S., \& McCarthy, E. P. (2009). Alternative mind-body therapies used by adults with medical conditions. Journal of Psychosomatic Research, 66(6), 511519.

Birnie, K., Speca, M., \& Carlson, L. E. (2010). Exploring selfcompassion and empathy in the context of mindfulness based stress reduction (MBSR). Stress and Health, 26(5), 359-371.

Bögels, S. M., Lehtonen, A., \& Restifo, K. (2010). Mindful parenting in mental health care. Mindfulness, 1(2), 107-120.

Caldwell, K., Harrison, M., Adams, M., Quin, R. H., \& Greeson, J. (2010). Developing mindfulness in college students through movement based courses: Effects on self-regulatory selfefficacy, mood, stress, and sleep quality. Journal of American College Health, 58(5), 433-442.

Cohen, J. (1988). Statistical power analysis for the behavior sciences. Hillsdale, New Jersey: Lawrence Earlbaum Associates.

Cullen, M. (2011). Mindfulness-based interventions: An emerging phenomenon. Mindfulness, 2(3), 186-193.

Esch, T., Duckstein, J., Welke, J., Stefano, G. B., \& Braun, V. (2007). Mind/body technique for physiological and psychological stress reduction: Stress management via Tai Chi training-A pilot study. Medical Science Monitor, 13(11), CR488-CR497.

Faul, F., Erdfelder, E., Lang, A. G., \& Buchner, A. (2007). G*Power 3: A flexible statistical power analysis program for the social, behavioral, and biomedical sciences. Behavior Research Methods, $39(2), 175-191$.

Frantzis, B. K. (1998). The power of internal martial arts: Combat secrets of Ba Gua, Tai Chi, and Hsing-I. Berkley, CA: North Atlantic Books. 
Grossman, P., Niemann, L., Schmidt, S., \& Walach, H. (2004). Mindfulness-based stress reduction and health benefits: A meta-analysis. Journal of Psychosomatic Research, 57(1), $35-43$.

Gyllensten, A. L., Hui-Chan, C. W. Y., \& Tsang, W. W. N. (2010). Stability limits, single-leg jump, and body awareness in older Tai Chi practitioners. Archives of Physical Medicine and Rehabilitation, 91(2), 215-220.

Hofmann, S. G., Sawyer, A. T., Witt, A. A., \& Oh, D. (2010). The effect of mindfulness-based therapy on anxiety and depression: a meta-analytic review. Journal of Consulting and Clinical Psychology, 78(2), 169-183.

Hupfeld, J., \& Ruffieux, N. (2011). Validation of a german version of the self-compassion scale (SCS-D). Zeitschrift für Klinische Psychologie und Psychotherapie, 40(2), 115-123.

Jacobson, B. H., Chen, H., Cashel, C., \& Guerrero, L. (1997). The effect of T'ai Chi Chuan training on balance, kinaesthetic sense, and strength. Perceptual and Motor Skills, 84(1), 27-33.

Jahnke, R., Larkey, L., Rogers, C., Etnier, J., \& Lin, F. (2010). A comprehensive review of health benefits of Qigong and Tai Chi. American Journal of Health Promotion, 24(6), e1-e25.

Kabat-Zinn, J. (2003). Mindfulness-based interventions in context: Past, present, and future. Clinical Psychology, 10(2), 144-156.

Klein, P. J., \& Adams, W. D. (2004). Comprehensive therapeutic benefits of Taiji: A critical review. American Journal of Physical Medicine \& Rehabilitation, 83(9), 735-745.

Kohls, N., Sauer, S., \& Walach, H. (2009). Facets of mindfulnessresults of an online study investigating the Freiburg mindfulness inventory. Personality and Individual Differences, 46(2), 224 230.

Kuyken, W., Watkins, E., Holden, E., White, K., Taylor, R. S., Byford, S., et al. (2010). How does mindfulness-based cognitive therapy work? Behaviour Research and Therapy, 48(11), 1105-1112.

Michalsen, A. (2010). Stressed patients, stressed physicians and the need for mind-body medicine. Forschende Komplementärmedizin, 17(5), 237-239.

National Institutes of Health (2010). What is complementary and alternative medicine? Retrieved from http://nccam.nih.gov/ health/whatiscam/

Nedeljkovic, M., Ausfeld-Hafter, B., Streitberger, K., Seiler, R., \& Wirtz, P. H. (2012). Taiji practice attenuates psychobiological stress reactivity - A randomized controlled trial in healthy subjects. Psychoneuroendocrinology. doi:10.1016/j.psyneuen.2011.12.007.

Neff, K. D. (2003a). Self-compassion: An alternative conceptualization of a healthy attitude toward oneself. Self and Identity, 2, 85-102.

Neff, K. D. (2003b). Development and validation of a scale to measure selfcompassion. Self and Identity, 2, 223-250.
Neff, K. D. (2009). Self-compassion. In M. R. Leary \& R. H. Hoyle (Eds.), Handbook of individual differences in social behavior (pp. 561-573). New York: Guilford Press.

Ospina, M. B., Bond, K., Karkhaneh, M., Buscemi, N., Dryden, D. M., Barnes, V., et al. (2008). Clinical trials of meditation practices in health care: Characteristics and quality. Journal of Alternative and Complementary Medicine, 14(10), 1199-1213.

Praissman, S. (2008). Mindfulness-based stress reduction: A literature review and clinician's guide. Journal of the American Academy of Nurse Practitioners, 20(4), 212-216.

Robinson, R. (2006). Tai Chi for you-The comprehensive guide to Tai Chi at home for everybody. London: Duncan Braid Publishers.

Shapiro, S. L., Astin, J. A., Bishop, S. R., \& Cordova, M. (2005). Mindfulness based stress reduction for health care professionals: Results from a randomized trial. International Journal of Stress Management, 12(2), 164-176.

Uhlig, T., Fongen, C., Steen, E., Christie, A., \& Odegard, S. (2010). Exploring Tai Chi in rheumatoid arthritis: A quantitative and qualitative study. BMC Musculoskeletal Disorders, 11(43). doi:10.1186/1471-2474-11-43

Walach, H., Buchheld, N., Buttenmüller, V., Kleinknecht, N., \& Schmidt, S. (2006). Measuring mindfulness - The Freiburg Mindfulness Inventory (FMI). Personality and Individual Differences, 40(8), 1543-1555.

Wall, R. B. (2005). Tai Chi and mindfulness-based stress reduction in a Boston public middle school. Journal of Pediatric Health Care, 19(4), 230-237.

Wang, C. (2011). Taiji and rheumatic diseases. Rheumatic Diseases Clinics of North America, 37(1), 19-32.

Wang, C., Bannuru, R., Ramel, J., Kupelnick, B., Scott, T., \& Schmid, C.H. (2010). Tai Chi on psychological well-being: Systematic review and meta-analysis. BMC Complementary and Alternative Medicine, 10(23). doi:10.1186/1472-6882-10-23

Wayne, P. M., \& Kaptchuk, T. J. (2008a). Challenges inherent to T'ai Chi research: Part I-T'ai Chi as a complex multicomponent intervention. Journal of Alternative and Complementary Medicine, 14(1), 95-102.

Wayne, P. M., \& Kaptchuk, T. J. (2008b). Challenges inherent to T'ai Chi research: Part II-defining the intervention and optimal study design. Journal of Alternative and Complementary Medicine, 14(2), 191-197.

Wolf, S. L., Coogler, C., \& Xu, T. (1997). Exploring the basis for Tai Chi Chuan as a therapeutic exercise approach. Archives of Physical Medicine and Rehabilitation, 78(8), 886-892.

Zahn, W.L. (2009). The effects of Tai Chi Chuan on mindfulness, mood, and quality of life in adolescent girls. (Doctoral dissertation). Retrieved from Proquest Dissertations and Theses. (UMI 3324384). 